\title{
Integración de los modelos de gestión en la dirección de la investigación en instituciones técnicas y tecnológicas. ${ }^{1}$ Synthesis of the Management Models in the Administration of Technical and Technological Institutions.
}

\author{
Juan Diego Betancur Arias ${ }^{2}$ \\ ${ }^{1}$ Fundación Universitaria María Cano, Colombia.
}

Artículo recibido en el mes de Enero de 2014; artículo aceptado en el mes de Marzo de 2014

Citación del artículo: Betancur, J. (2014). Integración de los modelos de gestión en la dirección de la investigación en instituciones técnicas y tecnológicas. I+D Revista de Investigaciones, 3(1), 24-29.

\section{Resumen}

En Colombia, la Ley General de Educación Superior, establece que todas las instituciones deben adelantar procesos de investigación, independientemente del carácter que tenga la institución o de los programas que posea; sin embargo, las instituciones técnicas y tecnológicas presentan dificultades al respecto. En este estudio descriptivo transversal se propone la articulación de los modelos de gestión del talento humano, la gestión de la tecnología y la gestión del conocimiento, con la intención de mostrar una alternativa que permita conseguir los logros y metas que se exigen en materia de investigación, para todas las instituciones de Educación Superior en Colombia.
Palabras clave: investigación, modelos de gestión, formación técnica y tecnológica, gestión del talento humano.

\section{Abstract}

Higher Education General Law in Colombia establishes that every institutions of this educational level must carry out investigation as its own substantial functions, even if they were technical, technological or universities; however in the case of these two first, there are some troubles about. In this cross-descriptive study a synthesis is proposed among: human talent management models, the technology management and the knowledge management in order to obtain a new choice to achieve the goals

\footnotetext{
${ }^{1}$ Artículo de investigación científica, de enfoque cuantitativo, resultado de un proyecto de investigación en curso, desarrollado en el grupo de investigación Centro de Investigación y Desarrollo Empresarial CIDE y fue financiado por la Fundación Universitaria María Cano (Colombia). Dirección: Calle 56 No. 41-90, PBX: (4)4025500, Medellín (Colombia).

${ }^{2}$ Psicólogo, Universidad de San Buenaventura, Medellín (Colombia). Doctorado en Psicología con Orientación en Neurociencia Cognitiva Aplicada, Universidad Maimónides, Buenos Aires (Argentina). Docente - Investigador Coordinador del Centro de Investigación y Desarrollo Empresarial CIDE, Fundación Universitaria María Cano (Colombia)
} 
Integración de los modelos de gestión en la dirección de la investigación en instituciones técnicas y tecnológicas.

demanded in the investigation field in the higher education institutions in Colombia.

Keywords: investigation, management models, technical education, technological information, human talent administration.

\section{Introducción}

La investigación como factor orientado a la creación, desarrollo y comprobación de conocimientos y técnicas, ocupa un ámbito de desarrollo de las funciones sustantivas de los entes de la Educación Superior. En el nivel técnico profesional y tecnológico, se orienta a la creación y adaptación de tecnologías (Ley 80 de 1980 reformada por la ley 30 de 1992; Ley 749 de 2002; Decreto 1295). No obstante, siguen existiendo dificultades en la adherencia de estas instituciones a las políticas estatales de ciencia, tecnología e innovación en lo relacionado con la función investigativa, afectando los procesos para el cumplimiento de las condiciones mínimas de calidad en la acreditación de los programas.

La gestión del conocimiento es una tarea que atañe a la formación tecnológica y técnica, aunque su orientación sea privilegiadamente la solución de problemas específicos del orden práctico en cada campo de actuación. Esta gestión requiere sistematicidad en el plano pedagógico y en la política de las instituciones educativas (Almashari, 2002) aunque sea una tarea compleja y difícil (Gold et al., 2001), la cual se realiza mejor en cuanto la comunicación sea más lateralizada (Hedlund, 1994). El conocimiento es el insumo base de la creación y se transfiere de manera permanente, se aplica y se reevalúa constantemente en función de los procesos de aprendizaje que se desarrollan en las instituciones educativas, pero su funcionamiento en ellas hace que se vuelva necesaria su gestión (Alavi \& Leidner, 2001), para que cumpla sus propósitos y realice sus etapas creativa, transferencial y aplicativa (Pedraja-Rejas et al., 2006).

La gestión del conocimiento, así entendida, requiere de una estrategia que la integre con los planes, políticas y acciones institucionales (Ramos y Ruiz 2004), y debe tener un carácter singular (Hamel \& Prahalad, 2005) que guíe la toma de decisiones (Nutt, 2000). Sin embargo, son personas quienes ejecutan estas decisiones y las que elaboran conocimiento dentro de las instituciones educativas, ya sean estudiantes, docentes o directivos, entre otras, por lo cual, el talento humano no debe desconocerse en la gestión del conocimiento, el cual está dirigido hacia la innovación y la solución de diferentes problemáticas según el campo tecnológico del cual se hable.

Desde esta perspectiva, se hace necesaria la comprensión de que la investigación científica y el desarrollo tecnológico en las instituciones en el nivel técnico profesional - tecnológico y centros de investigación son en la actualidad una base primordial para el crecimiento de la educación superior y la generación de talento humano de alta calidad. Es necesario incorporar, primeramente, los conocimientos adquiridos en la investigación al proceso de enseñanza aprendizaje, que coadyuve al mejoramiento de la calidad educativa. 
La Investigación, desarrollo, innovación y gestión tecnológica, poseen un valor estratégico tanto económico como educativo en cualquier institución de educación superior y son fundamentales para el desarrollo integral del país. En concordancia con las tendencias mundiales de agrupación de saberes en busca de la producción sistemática y válida de conocimiento, las instituciones técnicas y tecnológicas deben orientar sus esfuerzos hacia la organización de una estructura administrativa que facilite la conformación de grupos de investigación, en los cuales confluyan los esfuerzos y experiencias individuales de los docentes y el interés científico de los estudiantes, en la búsqueda del desarrollo sistemático y organizado de las actividades de investigación, subordinadas a un plan estratégico de grupo, que permita impactar aspectos de orden científico, técnico, social y ambiental. Son, por tanto, los grupos de investigación, multidisciplinarios en su mayoría, el escenario propio para la generación de ideas de investigación, la formación de semilleros de investigación, la producción científica y la divulgación de la ciencia. Lo anterior, soportado desde la configuración estructural que se propone, teniendo como referencia los modelos de gestión investigativa, elegidos para este fin, modelo de gestión del talento humano, gestión de la tecnología y gestión del conocimiento.

Integrar los modelos de gestión investigativa, gestión del talento humano, gestión de la tecnología y gestión del conocimiento en el direccionamiento estratégico de la investigación, en las instituciones de educación superior en el nivel técnico profesional y tecnológico en Colombia, se constituye en una alternativa frente a los procesos de investigación; con base en lo anterior, el presente estudio gira en torno a la pregunta acerca de ¿Qué dimensiones, relaciones, contenidos, funciones deben constituir un modelo de dirección de la investigación en instituciones técnicas y tecnológicas en el país?.

\section{Método}

\section{Tipo de estudio}

Se siguió un tipo de investigación no experimental descriptiva transversal de caso único, en el cual se describieron sucesos y eventos, tratando de comprender cómo se manifiesta el fenómeno de la actividad investigativa en las instituciones de Educación Superior en el nivel técnico profesional tecnológico y cómo los modelos de administración de la investigación que poseen estas instituciones en la actualidad, se pudiesen articular al modelo propuesto estratégicamente desde los modelos de gestión investigativa en la presente investigación. Fue estudio de caso único, dado que se buscaba la comprensión de un escenario investigativo concreto, en un tópico puntual, como es la administración y la gestión de la actividad investigativa en las instituciones de Educación técnico profesional y tecnológico; no se pretendía hacer predicciones sobre el mismo, la relación investigadores - investigado se centró desde un sistema de relaciones complejas holísticas, contemplado en la observación, el discurso y la participación.

\section{Participantes}

El tipo de ejercicio investigativo no requirió la elección de un universo poblacional y diseño 
Integración de los modelos de gestión en la dirección de la investigación en instituciones técnicas y tecnológicas.

muestral, dadas las características naturales del paradigma de investigación cualitativa que se utilizó para desarrollar la investigación.

Delimitación del caso único para el ejercicio investigativo: Se eligió una institución de Educación superior en el nivel de formación técnica profesional y tecnológica, cuyas características institucionales estuviesen reglamentadas por los factores y requisitos expuestos en las políticas nacionales e internacionales para este tipo de instituciones con el carácter expuesto. Además, se requirió conocer todo la estrategia investigativa, desde la formación en investigación e investigación formativa que se desarrolla en estas instituciones, con la intención de interpretar y comprender la dinámica para la construcción y formación integral de los técnicos y tecnólogos.

\section{Materiales e instrumentos}

entrevistas y observación directa.

Se realizaron diferentes entrevistas con actores educativos de los diferentes niveles en las instituciones y se observó de primera mano situaciones relacionadas con los aspectos de indagación.

\section{Procedimiento}

Se elaboró un diseño de las preguntas a realizar y funcionarios e instituciones a contactar. Seguidamente se planeó el recorrido de visitas para entrevistar y observar las variables de estudio y finalmente se recopilaron y analizaron los datos obtenidos.

\section{Resultados}

El tema de la investigación se encuentra en un círculo vicioso que limita sus posibilidades de éxito. Como resultado, se sigue constatando el pobre nivel de producción asociado a los proyectos de investigación. Esto constituye un grave problema. Como una contribución a superar este problema, se proponen alternativas de solución como: 1) Trabajar en la organización de la investigación institucional. 2) Generar políticas y normatividad que contribuyan al apoyo de la investigación. 3) Formas de organización que garanticen un adecuado proceso de vinculación y gestión tecnológica, de talento humano y conocimiento asociado a la investigación.

La integración y utilización de los modelos de gestión investigativa soportados desde los tres componentes estratégicos: gestión del talento humano, gestión del conocimiento y gestión de la tecnología, permiten a las instituciones de educación superior en el nivel técnico profesional y tecnológico, acciones efectivas para el cumplimiento de la cadena real de valor, además de poder desarrollar la libertad institucional con plena autonomía, según los principios de la formación en el ámbito de la educación superior para Colombia.

\section{Discusión}

Los resultados obtenidos, permiten señalar que un modelo integrado de gestión del conocimiento, de la tecnología y del talento 
humano, pueden responder de manera eficaz para el funcionamiento igualmente eficaz de la investigación en las instituciones técnicas y tecnológicas en el país.

\section{Referencias}

Alavi, M. \& Leidner, D. (2001). Knowledge Management and Knowledge Management Systems: Conceptual Foundations and Research Issues. MIS Quarterly 25: 107-136.

Al-Mashari, M. (2002). Business process management - a mandatory approach. Business Process Management Journal, 8(1),1-3.

Gold, A. H., Malhotra, A., \& Segars, A. H. (2001). Knowledge Management: An Organizational Capabilities Perspective. Journal of Management Information System, 18 (1) 185 214.

Hamel, G. \& C. K. Prahalad (2005). Propósito estratégico. En: Harvard Business Review América Latina, Clásicos HBRLA, 10-24.

Hedlund, G. (1994): A model of knowledge management and the $\mathrm{N}$-form Corporation. Strategic Management Journal, 5, 73-90.

Nutt, P. (2000) Decision-Making Success in Public, Private, and Third sector Organizations: Finding Sector Dependent Best Practice. Journal of Management Studies, 37, 77-108.

Pedraja-Rejas, L., Rodríguez-Ponce, E., Rodríguez-Ponce, J. (2006) Sociedad del conocimiento y dirección estratégica: Una propuesta integradora. Interciencia 31:570-576. 